\title{
Obstructed labour caused by foetal congenital hydronephrosis. Trans-abdominal drainage of the hydronephrosis with resultant delivery per vagina
}

\author{
Ishaq Funsho Abdul ${ }^{1}$, Abiodun S. Adeniran² Enoch U. Okpara², Olalekan Ibukun Oyinloye ${ }^{3}$, \\ Aisha Oluwatoyin Saka ${ }^{4}$, Emmanuel A. Adekanye ${ }^{5}$, Muhammed Jimoh Saka ${ }^{6}$, Abiodun Abdul G. Jimoh ${ }^{5}$ \\ ${ }^{1}$ Women's Health Attention Focus (WHAF), Kosemani Hospital, Ilorin, Nigeria \\ ${ }^{2}$ Department of Obstetrics and Gynaecology, University of Ilorin Teaching Hospital, Ilorin, Nigeria \\ ${ }^{3}$ Department of Radiology, University of Ilorin Teaching Hospital, Ilorin, Nigeria \\ ${ }^{4}$ Department of Pediatrics, University of Ilorin Teaching Hospital, Ilorin, Nigeria \\ ${ }^{5}$ Department of Obstetrics and Gynaecology, University of Ilorin Teaching Hospital, Ilorin, Nigeria \\ ${ }^{6}$ Department of Epidemiology and Community Health, University of Ilorin Teaching Hospital, Ilorin, Nigeria \\ Email: abdula_dr@yahoo.com
}

Received 23 October 2012; revised 25 November 2012; accepted 4 December 2012

\begin{abstract}
A case of a 25 year old $\mathrm{P}_{3}^{+0} 2$ alive Patient, with obstructed labour with fetal death and delivery of the fetus up to the trunk caused by congenital Bilateral Hydronephrosis is presented. Obstetric examination on admission supported by Abdominal Ultrasound revealed Bilateral Hydronephrosis which was then drained per abdomen using needle and intravenous fluid giving set with eventual relief of the obstruction and vaginal delivery of the stillborn baby.
\end{abstract}

Keywords: Obstructed Labour; Congenital Hydronephrosis; Trans-Abdominal Drainage; Vaginal Delivery; Developing Countries

\section{INTRODUCTION}

Though obstructed labour is eradicated or banished from developed Countries, in Nigeria like most developing Countries, obstructed labour with a dead Foetus is a reality and a well known cause of Maternal Morbidity and Mortality. The common causes of obstructed labour including contracted pelvic, uterine abnormalities, maternal pelvic tumours, fetal malpresentation, and congenital fetal abnormalities like Hydrocephalus, Polycystic Kidneys/Hydronephrosis or locked twins [1-4].

Even though congenital abnormalities of the Urinary Tract are well known causes of obstructed labour the fact that this particular patient was unbooked, presenting with the fetal head and hands already delivered and initially masquerading like shoulder Dystocia until ultrasound confirmation of Polycystic Kidney (Hydronephrosis) makes the case worthy of note.
The commonest treatment option for obstructed labour is Caesarean Section especially when the Foetus is alive, but is associated with a high post operative infection rate [1-3]. Destructive Operation such as decapitation, Cleidotomy with or without Embryotomy (Evisceration) when the Foetus is dead carries with it risk of maternal genital injuries, haemaorrhage as well as infection. The fact that Trans-abdominal drainage was able to decompress the kidney allowing for subsequent vaginal delivery (a form of Embryotomy) should draw the attention of practitioners to this safe method and possibly apply same in similar situations especially in developing countries where women have aversion for Caesarean section and inability to pay hospital bills following abdominal deliveries is a common occurrence.

\section{CASE REPORT}

The patient was Mrs. H.A., an unbooked Para $3^{+0}$ [2 alive] woman who was referred to our Labour ward from a private clinic as a case of obstructed labour from shoulder impaction. Mrs. H.A. attended Antenatal care in a herbal home and attempted delivery there at commencement of labour, but progress of labour was arrested after delivery of the baby up to the trunk necessitating her referral to the private clinic from where she was subsequently referred to the labour ward of the University of Ilorin Teaching hospital (UITH) for lack of expertise and equipment at the private hospital.

The Antenatal period was said have been uneventful and Mrs. H.A. said she visited the herbal home regularly until onset of labour at term. The Labour was said to have progressed well and she felt the urge to bear down just after three (3) hours of admission at the herbal home but that the baby could not be delivered after over thirty 
minutes despite the Fetal head haven been delivered.

Physical examination on admission revealed an anxious young woman in distress, not pale, not jaundiced but was febrile (Temperature was $38.0^{\circ} \mathrm{C}$ ). Mrs. H.A. was mildly dehydrated and her Pulse rate was 100 beats per minute and Blood Pressure was 130/80 mmHg. Both Heart sounds were normal and the Chest was clear clinically.

The Abdomen moved with respiration, no area of tenderness. Liver, Spleen and Kidneys were not palpably enlarged. The urinary Bladder was full and had to be drained with an indwelling catheter, the Fundal Height was 36 weeks palpable, not tender. The Foetal heart was not heard even with Sonicaid. The Foetal Limbs were palpable with rather roundish Trunk, the presentation was not felt as the Head and the two upper limbs were already in the perineum as confirmed on vaginal examination.

There was slight Vulva Edema and the fetal Head and Hands were visible at the Perineum. There was no masses in the vagina and the cervix was fully dilated with the Shoulders still at $0-1$ station. The Hands probably prolapsed or were forcibly brought down at the herbal home (Figure 1).

The admitting house officer and Residents thought of Shoulder Dystocia as a cause of the obstructed Labour with intrauterine Fetal Death.

The plan then was to prepare for Destructive Operation or Caesarean Section if there is no Skilled Hand to perform the Destructive Operation. Further review then requested for an Ultrasound scan to ascertain the nature of the suspected Fetal Abdominal Mass; Ultrasound revealed bilateral enlarged kidneys, with

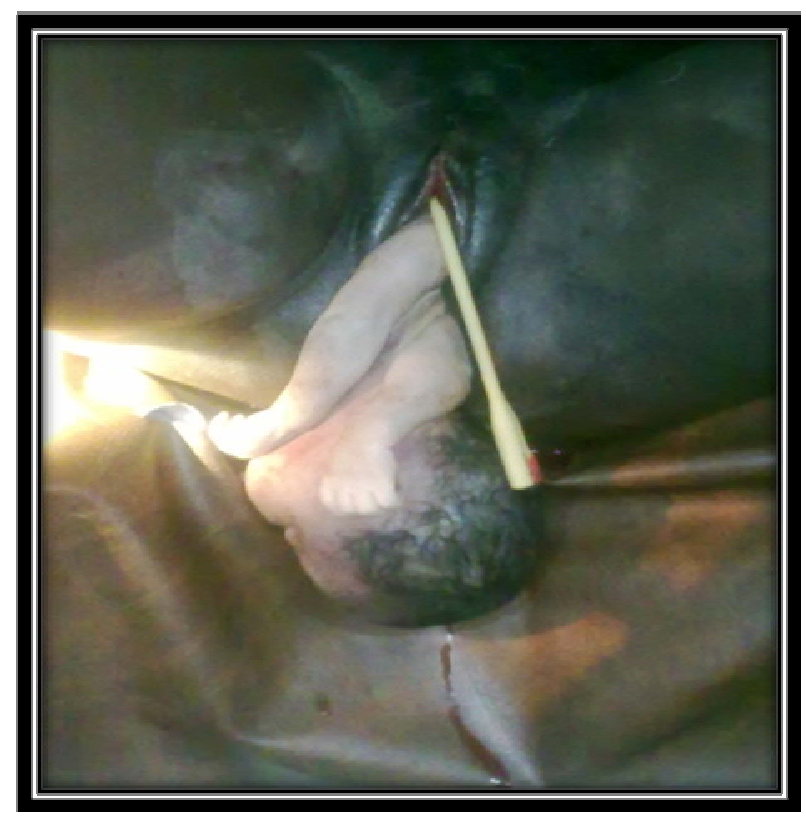

Figure 1. Foetal head and hands at the perineum. marked dilatation of the calyces and thinning of the renal parenchyma. The urinary bladder was also distended and associated reduced amniotic fluid in a male foetus. An Ultrasound diagnosis of Bilateral Hydronephrosis possibly from a Posterior Urethral Valve (PUV) was made and confirmed by the Consultant Sonologist (Figure 2).

A Diagnosis of Obstructed Labour secondary to Congenital Hydronephrosis was then made and informed Consent was obtained to drain the Hydronephrosis per abdomen under Ultrasound guide after the patient was counseled on management options.

The Abdominal Wall was aseptically prepared at the point where the Hydronephrosis were closest to the Maternal Abdominal Wall with no intervening bowel loops. Intravenous Fluid Giving Set with its $18 \mathrm{G}$ needle was directed at the Hydronephrosis and about 650 milliliters of urine was collected (Figure 3). There was subsequently reduction in the Fundal Height and gentle pushing by the patient assisted by the attending Obstetrician completed the delivery of the shoulders and the rest of the stillborn Male Baby weighing $2.8 \mathrm{Kg}$. Intramuscular $0.5 \mathrm{mg}$ Ergometrine was given and Oxytocin infusion 40 units in 500 milliliters of $5 \%$ Dextrose Water were set up to run for 8 Hours. The patient had earlier being rehydrated, started on triple Antibiotics regiment of Intravenous Metronidazole 500 mg 8 hourly, intravenous gentamycin 80 mg 12 hourly and intravenous Ampiclox $500 \mathrm{mg} 6$ hourly. This was continued for 48 hours after which the Ampiclox and

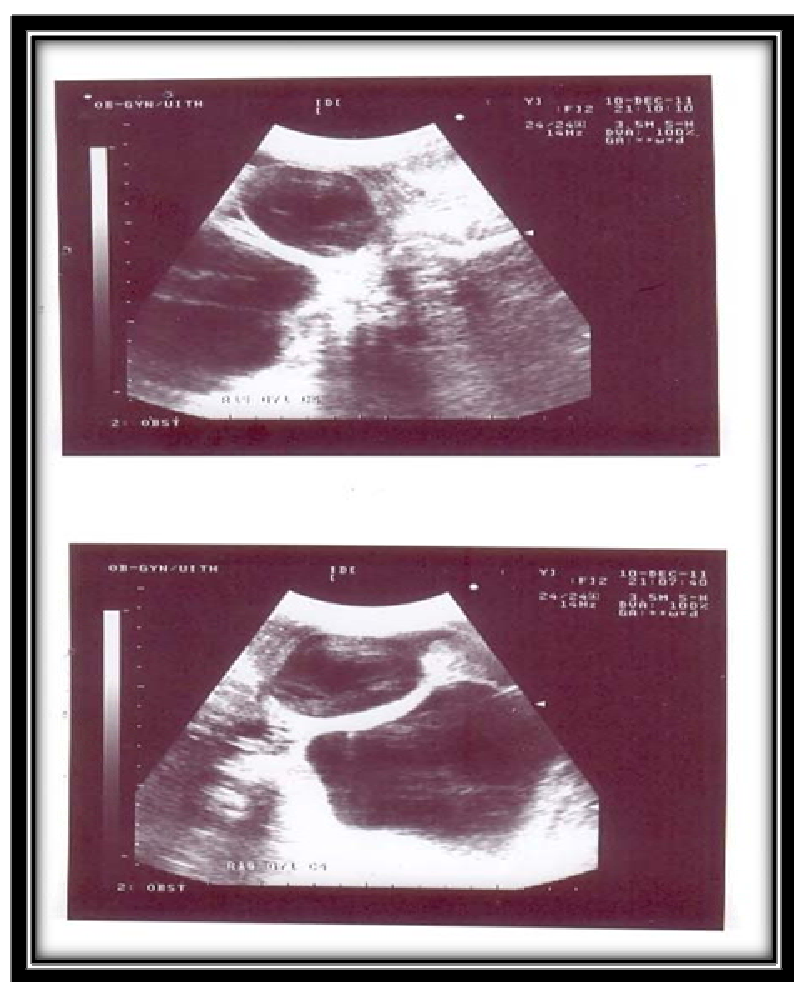

Figure 2. Ultrasound picture of the hydronephrosis. 


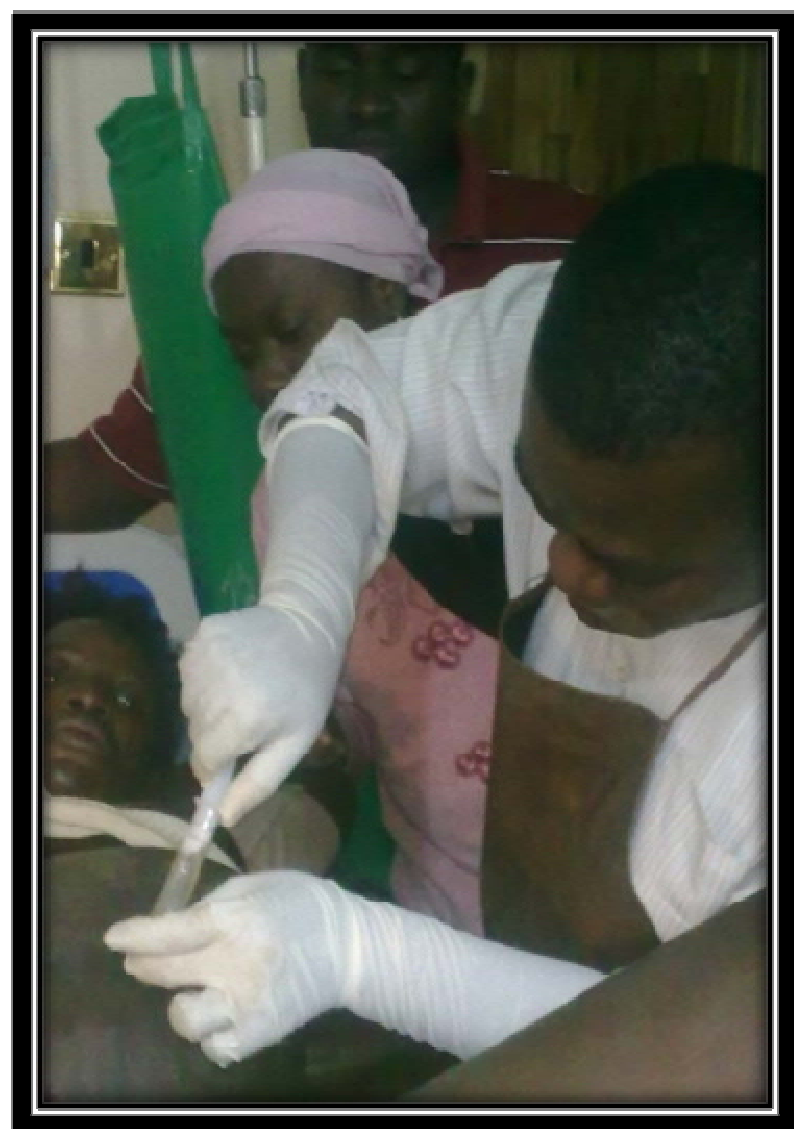

Figure 3. Aseptic Transabdominal drainage.

metronidazole were changed to oral preparations for the next five days and the gentamycin continued till fifth day postpartum.

Mrs. H. A refused to sign consent for post mortem examination and the relations took the baby away for burial. The Patient's post delivery condition was stable and she was discharged on above drugs. She was asked to come for post natal visit in six weeks.

\section{DISCUSSION}

The reported incidence of obstructed labour in this hospital was 3.2\% [1] which is very much in line with reported incidences in Nigeria and other parts of the developing world [2-6]. Foeto-pelvic disproportion was the commonest cause in this centre and Foetal congenital anomaly as a cause of obstructed labour was only $1.5 \%$ of cases with Hydrocephalus been the commonest; but this is the first time that bilateral Hydronephrosis will be documented.

Congenital hydronephrosis is a congenital anomaly which is easily detected on ultrasonography during pregnancy and appropriate management plan which can be expectant management, prenatal or post natal surgical treatment can be formulated. Obstructive and bilateral lesions are more threatening than unilateral and non obstructive lesions with oligohydramnios being the best predictor of outcome in bilateral obstructive cases[7] and are an acceptable indication for in-utero surgical decompression [8]. To date, no medical interventions are indicated for the treatment of antenatal Hydronephrosis except the administration of glucocorticoids to mothers whose fetuses have developed oligohydramnios and resultant pulmonary hypoplasia to stimulate production of surfactant associated proteins and enhance lung maturation [9]. The fact that this patient could still present in the $20^{\text {th }}$ century without contact at any Antenatal Clinic shows that lack of access to basic (Primary healthcare) is still prevalent in our environment and where present, extreme poverty remains a major hindrance to patronage of such health facilities.

Proper Antenatal care would have led to early detection of the congenital bilateral hydronephrosis at a routine Ultrasound scan or on request at clinical suspicion and the baby managed appropriately.

Assisted Vaginal Delivery such as Forceps or vacuum was not appropriate in this case because the baby was already dead and more importantly because Labour was obstructed anyway by the Foetal trunk. Therefore a destructive operation such as Embryotomy (Evisceration) after Decapitation and or Cleidotomy would have been treatment of choice. Caesarean Section has often been the alternative in Centres where destructive operations are not in use or of limited experience though abdominal deliveries in such cases carries higher risk of post operative wound infection as high as $34.3 \%$ [1-3]. However, destructive operations poses a substantial risk to the Mother in terms of Genital Tract lacerations or injuries to surrounding structures such as bladder or lower gastro intestinal tract [1-6].

The treatment method used here which is Trans abdominal drainage of the Hydronephrosis is also a form of Embryotomy which is cheaper and safer. This option can therefore be considered in selected cases similar to the one presented under ultrasound guidance like was done in this instance. There is an ultrasound machine with biconvex probe stationed permanently in the labour room in this centre.

The general care of the patient in this case was minimal because Mrs. H. A was in relatively stable clinical condition on arrival with mild dehydration and a high risk for sepsis necessitation triple Antibiotics immediately after admission.

Cases of obstructed labour sometimes need blood transfusion and extensive preoperative preparation with Naso-gastric tube insertion to decompress the stomach and use of $\mathrm{H}_{2}$ receptor Antagonist or proton pump inhibitors to decrease Gastric acidity if general anesthesia was contemplated as prevention for Mendelson syndrome. All these were avoided in this instance as the patient only needed aseptic preparation of the needle 
puncture site and the procedure was aseptically carried out; this avoidance of surgery eliminated the occurrence of wound infection following caesarean section.

The only likely risk in this procedure has to do with the Needle puncture site on the Uterus which usually heals primarily. There is a likelihood of the needle site becoming a weak point for uterine Laceration in the future during parturition, but it is really a remote possibility, after all there is a long old practice of direct injection of Oxytocics directly to the Uterus in cases of Post Partum Haemorrhage due to Uterineatony [10]. The patient has however been counseled to book in the hospital in subsequent pregnancy and Labour for proper monitoring because of this.

In addition, we ensured that no bowel loop was in the line of the procedure as prevention for bowel injury.

The high point of this case presentation is to show that lack of access to genuine antenatal care is still prevalent in our society, emphasize the hindrance of assessing such due to abject poverty and also that obstructed labour is still common and practitioners must constantly evolve ways and means to provide safe, cheaper and effective treatment whenever such cases arise.

\section{ACKNOWLEDGEMENTS}

I use the medium to thank Mr. Oyeniyi Seyi of Women's Health Attention Focus (WHAF) for typing this manuscript.

\section{REFERENCES}

[1] Aboyeji, A.P. and Fawole, A.A. (1999) Obstructed labour in Ilorin, Nigeria: A one year prospective study. Nigerian Medical Practitioner, 38, 25-28.
[2] Nwogu-Ikojo, E.E., Nweze, S.O. and Ezegwui, H.U. (2008) Obstructed labour in Enugu Nigeria. Journal of Obstetrics \& Gynecology, 28, 596-599. doi:10.1080/01443610802281682

[3] Ozumba, B.C. and Uchegbu, H. (1991) Incidence and management of obstructed labour in eastern Nigeria. Australian and New Zealand Journal of Obstetrics and Gynaecology, 31, 213-216

[4] Mahedra, N.P. (2006) Destructive operations in obstetrics: Editorial. The Journal of Obstetrics \& Ganecology of India, 56, 113-114.

[5] Dolea, C. and AbouZahr, C. (2003) Global burden of obstructed labour in the year 2000. Global Burden of Disease 2000, WHO Press, Geneva.

[6] WHO (2008) Managing prolonged and obstructed labour: Education manual for teachers of midwifery. 2nd Edition, WHO Press, Geneva.

[7] Reznik, V.M., Murphy, J.L., Mendoza, S.A., et al. (1989) Follow up of infants with obstructive uropathy detected in utero and treated postnatally. Journal of Pediatric Surgery, 24, 1289-1292. doi:10.1016/S0022-3468(89)80569-X

[8] Harrison, M.R., Golbus, M.S., Filly, R.A., et al. (1982) Fetal surgery for congenital hydronephrosis. The New England Journal of Medicine, 306, 591-593. doi:10.1056/NEJM198203113061006

[9] Bolt, R.J., van Weissenbruch, M.M., Lafeber, H.N., et al. (2001) Glucocorticoids and lung development in the fetus and preterm infant. Pediatric Pulmonology, 32, 76-91. doi:10.1002/ppul.1092

[10] Anderson, H.F. and Hopkins, M.P. (2004) Postpartum haemorrhage. Gynaecology and Obstetrics, 1-6, Chapter 20. 